\title{
Fluidized Bed Drying Effect on the Aerogel Powder Synthesis
}

\author{
Seonghoon Hong, Dongkyu Lee, Changsup Oh*, and Yong-Ha Kim**† \\ Chungbuk National University, Chungbuk Cheongjoosi 361-763, Korea. \\ *Korea Institute of Science and Technology Information, Reseat program, Daejeon, 305-806, Korea \\ **Pukyong National University, Busan, 608-739, Korea
}

(Received 12 January 2012, Revised 24 February 2012, Accepted 24 February 2012)

\begin{abstract}
A fluidized bed drying approach was utilized to the synthesis of water glass based silica aerogel powders. The effects of the fluidized bed drying conditions such as the superficial velocity and temperature of hot air and bead size as well as bead/wet-gel ratio, on the physical properties such as tapping density and productivity of the aerogel powders were systematically investigated. The experimental results showed that the amount of beads mixed with wet-gels in the fluidized bed column has the most profound impact on the fluidization efficiency, greatly enhancing the yield of the aerogel powders up to $98 \%$ with a proper bead/wet-gel weight ratio as compared to $72 \%$ without using beads. No significant change was observed in the tapping density over a wide range of the fluidized drying condition. Consequently the fluidized bed drying approach shows a good promise as an alternative route for the large-scale production of the aerogel powders.
\end{abstract}

Key words : Fluidized bed drying, Silica aerogel, Water-glass, Tapping density

\section{Introduction}

Silica aerogels are nanostructured solids usually synthesized by sol-gel process. They consist of interconnected nanoparticle building, which form highly porous threedimensional silica network having many interesting properties such as high specific surface area (500-1000 $\left.\mathrm{m}^{2} / \mathrm{g}\right)$, low bulk density $\left(10-200 \mathrm{~kg} / \mathrm{m}^{3}\right)$ and low thermal conductivity $(\sim 0.02 \mathrm{~W} / \mathrm{mk})$ [1-3]. These feature have led the aerogels for various scientific and industrial applications such as super thermal insulation [4], radiation detectors [5] and adsorbent for waste treatment [6]. The aerogels have been synthesized in various forms such as monoliths, granules and power. In the synthesis of silica aerogels by ambient pressure drying, a wet-gel is dried in a furnace under controlled drying conditions to yield aerogel. This drying process however is not suitable for the large scale production of the aerogels in power form. This is because the aerogel powder particles, being extremely light weight, easily get spread in

${ }^{\dagger}$ To whom corresponding should be addressed. Pukyong National University, Busan, 608-739, Korea Tel./Fax : 042-869-0767; E-mail : yhkim@pknu.ac.kr the surrounding atmosphere which not only contaminates the air but also leads to the significant mass loss during the handling. Therefore, it is crucial to have a continuous and closed type of drying process for drying of the wet-gel slurry as far as the large scale production of powdered aerogels is concerned. The high production cost and long preparation time have also restricted the manufacturing process of the silica aerogels from towards the way of the scale-up for mass production [7]. Though several attempts have been made [8-11], recently some meaningful results. In our earlier work, a novel process has been developed to rapidly synthesize super-hydrophobic silica aerogel powders without losing their important characteristics by apply fluidzed bed technique to the drying step of the wet-gels [12].

In the present work, the controllable parameters of the fluidized bed such as, drying gas velocity and bed temperature as well as bead size and bead/wet-gel ratio are systematically manipulated and their effects on the physical properties and productivity of the synthesized aerogel powders are quantitatively analyzed. 


\section{Experimental}

Only brief description of our experimental procedure is given here since more details can be found elsewhere [12]. The wet-gels were prepared from the solgel polymerization of a commercially available waterglass precursor (Il Shin Co., Korea) by co-precursor method and then immersed in n-hexane for 3 hours so as to undergo one-step solvent exchange process. The wet-gels were subsequently fed into the fluidized bed, as shown in Fig. 1 and mixed with spherical glass beads for drying at an ambient pressure to obtain aerogel powders. The mixture of wet-gels and glass beads were maintained to approximately occupy one fourth of the fluidized bed column. The size of the glass bead was varied from $1 \times 10^{-4}$ to $6 \times 10^{-4} \mathrm{~m}$ to investigate its effect on the physical properties of the aerogel powders. The wet-gel slurry was fluidized and dried with hot air with various superficial gas velocities ranging from $0.03 \mathrm{Nm} / \mathrm{s}$ to $0.07 \mathrm{Nm} / \mathrm{s}$. The temperature of the air was also varied in the range of $180^{\circ} \mathrm{C}$ to 300 ${ }^{\circ} \mathrm{C}$. The aerogel powders discharged from the column were subsequently fluidized twice more and separately collected in Trap-I, Trap-II and Trap-III depending on their weights.

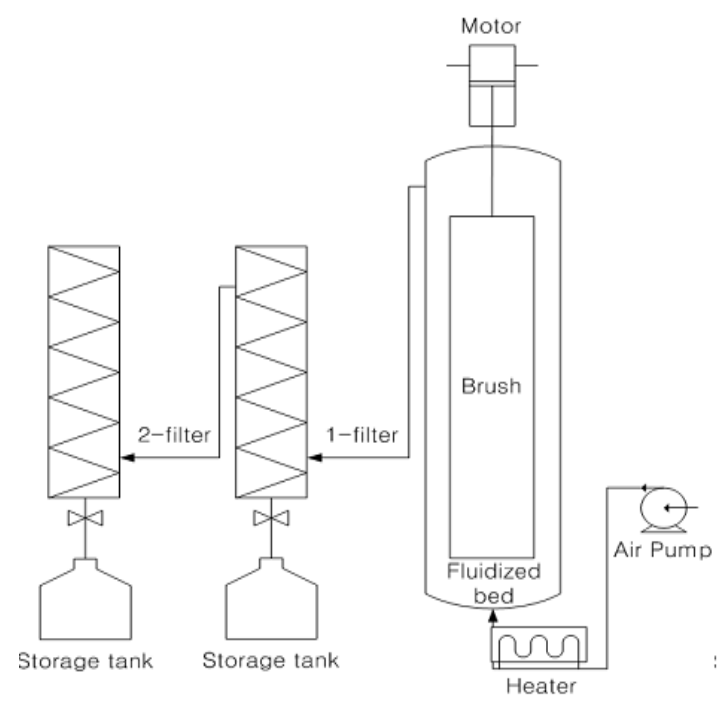

Fig. 1. Schematic representation of the experimental setup used for the fluidized bed drying of the aerogel powder.

\section{Results and Discussion}

To investigate the effects of fluidized bed drying conditions on the characteristics of the synthesized silica aerogel powders, the superficial velocity and temperature of the hot air and the bead size as well as the bead/gel weight ratio were systematically varied.

Fig. 2 shows the effects of the hot air conditions and the glass bead size on the tapping density of the silica aerogel powders. In any case, the tapping density of the dried aerogel powder remained almost unaffected around $0.09 \mathrm{~g} / \mathrm{cm}^{3}$ mainly because the structural evolution of the aerogel network was governed by the chemical composition of the starting materials and their synthetic condition used in the preparation of the wet-gel and was not greatly modified by the drying conditions. Though not affected significantly, the tapping density exhibited a tendency to slightly increase when the bead size increased, as shown in Fig. 2(c), simply because larger beads had the surface area of the wet-gel in the

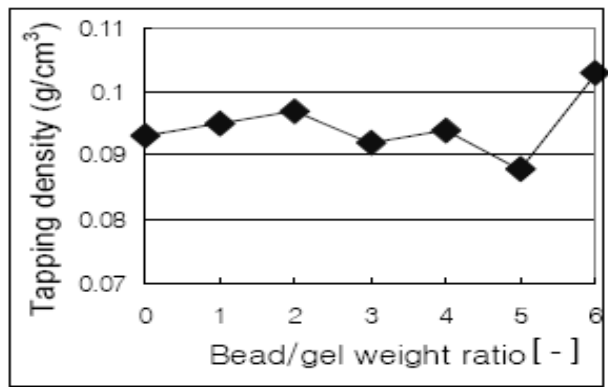

(a)

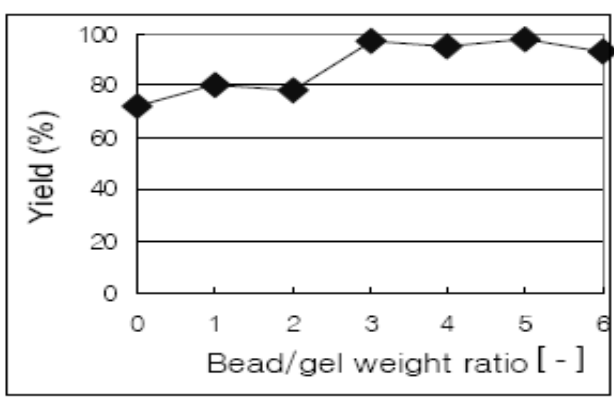

(b)

[Bead size: $0.2 \mathrm{~mm}$, Bed temperature: $200^{\circ} \mathrm{C}$, Superficial gas velocity: $3.3 \times 10^{-2} \mathrm{Nm} / \mathrm{s}$, ]

Fig. 2. Variations of tapping density and yield of aerogel powder as a function of bead/ gel weight ratio, respectively. 
drying column decreased.

Figs. 3(a) and (b) show the effects of the bead/gel weight ratio on the tapping density and yield of the aerogel powders respectively. The tapping density did not change significantly even when the bead/gel weight ratio was varied from 0 to 6 , confirming that the chemical composition of the starting materials and their synthetic condition predominantly determine the microstructure of the aerogel powders. However the yield of aerogel powders was significantly improved with the increased bead/gel weight ratio, as shown in Fig. 3(b).

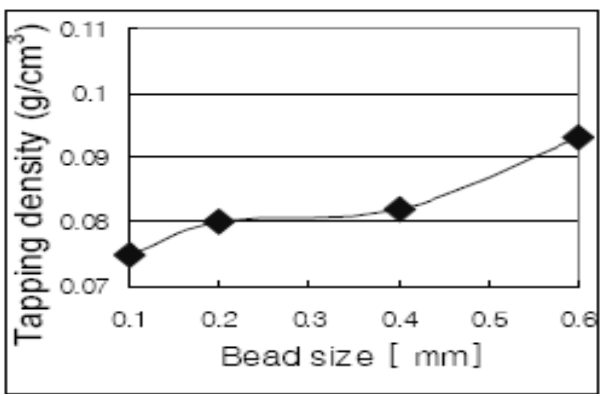

(a) Bead/gel weight ratio: 5, Bed temperature: $200^{\circ} \mathrm{C}$, Superficial gas velocity: $3.3 \times 10^{-2} \mathrm{Nm} / \mathrm{s}$

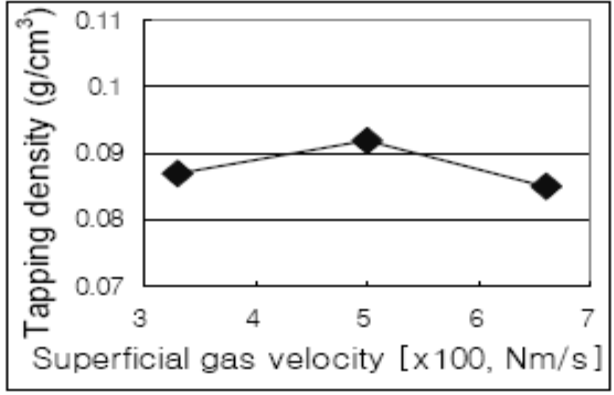

(b) Bed temperature: $200^{\circ} \mathrm{C}$, Bead size: $0.2 \mathrm{~mm} \mathrm{Bead} / \mathrm{gel}$ weight ratio: 5

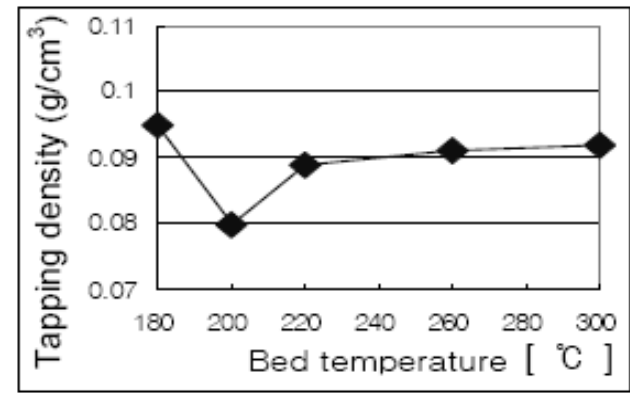

(c) Bead/gel weight ratio: 5, Bead size: $0.2 \mathrm{~mm}, \mathrm{Su}-$ perficial gas velocity: $3.3 \times 10^{-2} \mathrm{Nm} / \mathrm{s}$

Fig. 3. Effects of glass bead size, superficial gas velocity and fluidized bed temperature on the tapping density of the aerogel powder, respectively.
As the bead/gel weight ratio increased while the amount of the gel and the bead size were fixed, the number of the beads mixed with the wet-gels and consequently the surface area of the wet-gels exposed to the hot air increased, ultimately enhancing the productivity of the aerogel powders. Furthermore, when the bead/gel weight ratio was too small then it turned out difficult to break down the aggregates of the wet-gel and the aerogel powder particles as well, in turn forming too large
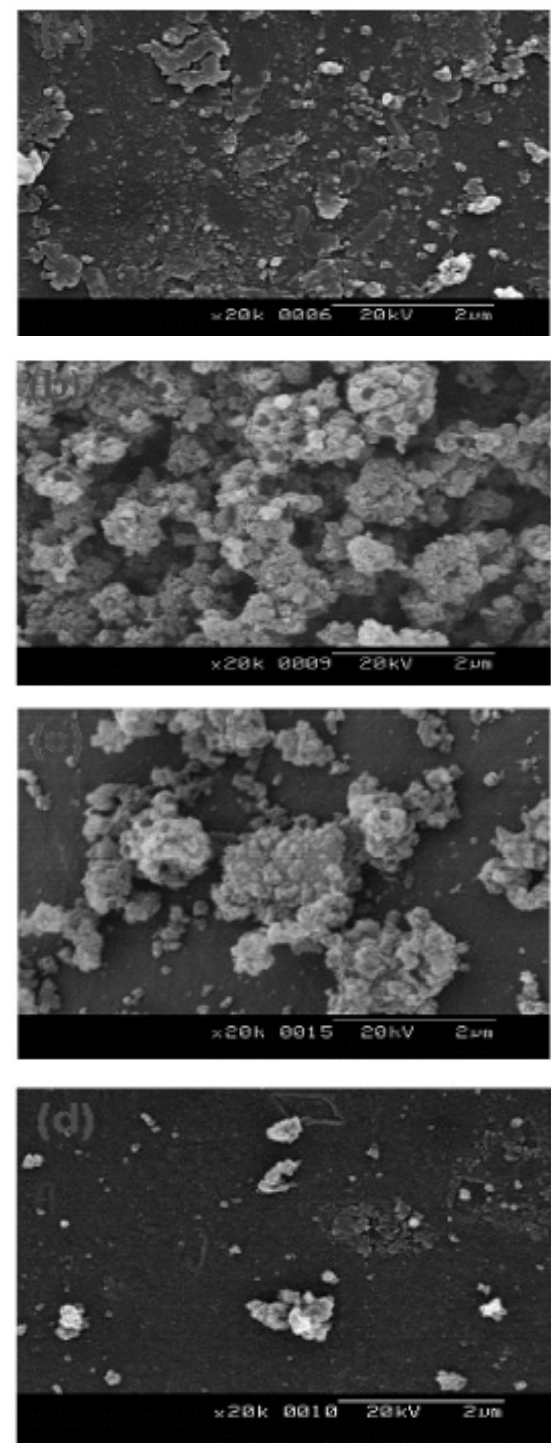

Fig. 4. SEM images of the glass bead: (a) Bead before use, (b) Bead coated with wet-gel slurry before drying, (c) Bead coated with the remaining aerogel powder after fluidized drying and (d) Bead after hexane-water treatment. 
agglomerates to be fluidized. It is worthwhile to note that the yield sharply increased from 79 to $98 \%$ when the bead/gel weight ratio increased from 2 to 3, implying that the proper fluidization of the wet-gel in the drying column can greatly enhance the yield of the aerogel powders.

Fig. 4 shows the SEM images of the glass beads (a) beads before use, (b) beads coated with the wet-gel slurry before drying, (c) beads coated with the residual aerogel powders after fluidized bed drying, and (d) beads after the hexane-water treatment. It can be clearly observed that the residual aerogel powders stuck to the beads after the drying could be effectively removed by the hexane-water treatment. Consequently it is possible to use the beads in a cyclic manner for the fluidized bed drying of the we-gels.

\section{Conclusions}

The fluidized bed drying conditions such as, the superficial velocity and temperature of the air and the bead size as well as the bead/gel weight ratio have been systematically varied to investigate their effects on the tapping density of the synthesized aerogel powders and the efficiency of the drying process. The results showed that the yield could be greatly enhanced by the proper fluidization of the bead/wet-gel mixture without significantly affecting the tapping density of the aerogel powders. The tapping density remained almost unaffected around $0.09 \mathrm{~g} / \mathrm{cm}^{3}$ over a wide range of drying conditions. Meanwhile the yield increased up to $98 \%$ by using the bead/wet-gel weight ratio greater than 3, showing a great potential for the application of the fluidized bed drying to the large-scale production of the aerogel powders.

\section{REFERENCES}

1. J. Fricke, T. M. Tilloston, Thi. Soli. Film., 297, 212 (1997).

2. A. P. Rao, A. V. Rao and G. M. Pajonk, J. Sol Gel Sci. and Tech., 36, 285 (2005).

3. C. S. Oh, Bhagat, S. D., Y. H. Kim, Y. S. Ahn and J. G. Yeo, Micro. Meso. Mater., 100, 350 (2007).

4. Y. H. Kim, Bhagat, S. D., Y. S. Ahn and J. G. Yeo, Micro. Meso. Mater., 96, 237 (2006).

5. Y. H. Kim, Bhagat, S. D., M. J. Moon, Y. S. Ahn and J. G. Yeo, Soli. Stat. Sci., 9, 628 (2007).

6. Y. H. Kim, Bhagat, S. D., G. B. Yi, Y. S Ahn, J. G. Yeo, Y. T. Choi, Micro. Meso. Mater., 108, 333 (2008).

7. Y. H. Kim, Bhagat, S. D., K. H. Suh, Y. S. Ahn, J. G. Yeo, J. H. Han, Micro. Meso. Mater., 112, 504 (2008)

8. K. T. Park, Bhagat, S. D., Y. H. Kim, J. S. Kim and J. H. Han, Soli. Stat. Sci., 11, 16 (2007). 